\title{
GROUPE DE BRAUER NON RAMIFIÉ DE QUOTIENTS PAR UN GROUPE FINI
}

\author{
J.-L. COLLIOT-THÉLÈNE \\ (Communicated by Lev Borisov)
}

\begin{abstract}
RÉSumÉ. Soit $k$ un corps, $G$ un groupe fini, $G \hookrightarrow S L_{n, k}$ un plongement. Pour $k$ algébriquement clos, Bogomolov a donné une formule pour le groupe de Brauer non ramifié du quotient $S L_{n, k} / G$. On examine ce que donne sa méthode sur un corps $k$ quelconque (de caractéristique nulle). Par cette méthode purement algébrique, on retrouve et étend des résultats obtenus au moyen de méthodes arithmétiques par Harari et par Demarche, comme la trivialité du groupe de Brauer non ramifié pour $k=\mathbf{Q}$ et $G$ d'ordre impair.

[Let $k$ be a field, $G$ a finite group, $G \hookrightarrow S L_{n, k}$ an embedding. For $k$ an algebraically closed field, Bogomolov gave a formula for the unramified Brauer group of the quotient $S L_{n, k} / G$. We develop his method over any characteristic zero field. This purely algebraic method enables us to recover and generalize results of Harari and of Demarche over number fields, such as the triviality of the unramified Brauer group for $k=\mathbf{Q}$ and $G$ of odd order.]
\end{abstract}

\section{INTRODUCTION}

Soient $k$ un corps de caractéristique zéro, $\bar{k}$ une clôture algébrique de $k$, et $\mathfrak{g}=\operatorname{Gal}(\bar{k} / k)$. À toute $k$-variété algébrique géométriquement intègre $W$, de corps des fonctions $k(W)$, on associe d'une part son groupe de Brauer cohomologique $\operatorname{Br}(W):=H_{\text {ét }}^{2}\left(W, \mathbb{G}_{m}\right)$, d'autre part le groupe de Brauer non ramifié $\operatorname{Br}_{n r}(k(W) / k)$ qu'on notera ici simplement $\mathrm{Br}_{n r}(k(W))$. Pour la définition et les propriétés de base de ces groupes, on renvoie à [8, 2] et [6]. Rappelons simplement que si $W$ est lisse sur $k$, alors la restriction au point générique induit une inclusion

$$
\operatorname{Br}(W) \hookrightarrow \operatorname{Br}(k(W))
$$

et que si $W$ est projective et lisse, alors, sous l'hypothèse car. $(k)=0$, cette inclusion induit un isomorphisme (cf. [2, (3.9)]):

$$
\operatorname{Br}(W) \stackrel{\sim}{\longrightarrow} \operatorname{Br}_{n r}(k(W)) .
$$

Soit $n \geq 2$ un entier, $X=S L_{n, k}$ et $G$ un groupe fini vu comme $k$-groupe constant, et $G \hookrightarrow S L_{n, k}$ un $k$-plongement de groupes. Notons $Y$ l'espace homogène quotient $X / G$. Le corps des fonctions $k(Y)$ de $Y$ est le corps $k(X)^{G}$ des invariants du corps des fonctions $k(X)$ sous l'action de $G$. L'extension de corps $k(X) / k(Y)$ est galoisienne de groupe $G$.

Ces notations seront conservées dans tout l'article.

Received by the editors January 25, 2012 and, in revised form, March 23, 2012; April 17, 2012; May 7, 2012; and May 11, 2012.

2010 Mathematics Subject Classification. Primary 12G05, 14E08, 14F22, 14M20. 
On se propose d'examiner le groupe de Brauer non ramifié $\mathrm{Br}_{n r}(k(Y))$. Comme rappelé ci-dessous (lemme 2.1), ce groupe (à isomorphisme non unique près) ne dépend que de $G$, il ne dépend ni de $n$ ni du plongement $G \hookrightarrow S L_{n, k}$.

Comme $Y$ est une $k$-variété lisse intègre, on a $\operatorname{Br}_{n r}(k(Y)) \subset \operatorname{Br}(Y)$.

On va suivre ici la méthode purement algébrique qu'avait utilisée Bogomolov ([1], [6, Thm. 7.1]) lorsque $k$ est algébriquement clos.

Ceci permet d'étendre au groupe $\mathrm{Br}_{n r}(k(Y))$ tout entier certains des résultats établis par des méthodes arithmétiques par Harari [9] et par Demarche [7] sur le sous-groupe "algébrique"

$$
\operatorname{Br}_{n r, 1}(k(Y)):=\operatorname{Ker}\left[\operatorname{Br}_{n r}(k(Y)) \rightarrow \operatorname{Br}(\bar{k}(Y))\right],
$$

sous-groupe formé des classes qui s'annulent par passage au corps des fonctions $\bar{k}(Y)$ de $\bar{Y}=Y \times_{k} \bar{k}$.

En particulier, on montre ici (Corollaire 5.7) que pour tout corps $k$ de caractéristique zéro ne possédant qu'un nombre fini de racines de l'unité, pour le quotient $Y=S L_{n, k} / G$ avec $G$ fini constant d'ordre premier au cardinal du groupe des racines de l'unité dans $k$, on a $\operatorname{Br}(k)=\operatorname{Br}_{n r}(k(Y))$.

Dans tout cet article, on suppose le corps de base $k$ de caractéristique zéro. Un grand nombre des résultats vaut sur un corps de base quelconque, si l'on se limite à considérer des groupes finis $G$ d'ordre premier à la caractéristique. En caractéristique non nulle, pour de tels groupes, la condition $\operatorname{Cyc}(G, k)$ ci-dessous est automatiquement satisfaite.

Je remercie le rapporteur pour sa lecture attentive du tapuscrit.

\section{Notations et RAPpels}

Pour tout premier $p$ impair et tout entier $r \geq 1$, l'extension $k\left(\mu_{p^{r}}\right) / k$ est cyclique. Il en est encore ainsi pour $p=2$ si -1 est un carré dans $k$.

On note $\operatorname{Cyc}(G, k)$ la condition:

Pour tout sous-groupe cyclique $\mathbf{Z} / 2^{r} \subset G$, l'extension $k\left(\mu_{2^{r}}\right) / k$ est cyclique.

Pour $A$ un groupe abélien, $n>0$ un entier et $l$ un nombre premier, on note $A[n] \subset A$ le sous-groupe annulé par $n$ et on note $A\{l\} \subset A$ le sous-groupe de torsion $l$-primaire.

Un $k$-groupe algébrique linéaire connexe $H$ est dit spécial si pour toute $k$-variété $X$ tout torseur sur $X$ sous $H$ est localement trivial pour la topologie de Zariski. Les groupes linéaires $G L_{m, k}$, les groupes spéciaux linéaires $S L_{n, k}$ et les produits de tels groupes sont spéciaux. Ce sont en outre des $k$-groupes $k$-rationnels: la $k$-variété sous-jacente est $k$-birationnelle à un espace projectif.

On dit que deux $k$-variétés géométriquement intègres $U$ et $V$ sont stablement $k$-birationnellement équivalentes s'il existe des espaces projectifs $\mathbf{P}_{k}^{r}$ et $\mathbf{P}_{k}^{s}$ tels que $U \times{ }_{k} \mathbf{P}_{k}^{r}$ est $k$-birationnel à $V \times_{k} \mathbf{P}_{k}^{s}$. Pour deux telles $k$-variétés $U$ et $V$, on a $\operatorname{Br}_{n r}(k(U)) \simeq \mathrm{Br}_{n r}(k(V))$ [6, Prop. 5.7]).

Lemme 2.1. Soit $G$ un groupe fini. Si l'on a des plongements de $k$-groupes $G \hookrightarrow H_{1}$ et $G \hookrightarrow H_{2}$ où $H_{1}$ et $H_{2}$ sont des $k$-groupes algébriques spéciaux $k$-rationnels, alors:

(i) Les $k$-variétés $H_{1} / G$ et $H_{2} / G$ sont stablement $k$-birationnellement équivalentes.

(ii) $\operatorname{Br}_{n r}\left(k\left(H_{1} / G\right)\right) \simeq \operatorname{Br}_{n r}\left(k\left(H_{2} / G\right)\right)$.

Démonstration. L'énoncé (i), variante du "lemme sans nom" est établi dans 6, Prop. 4.9]. L'énoncé (ii) résulte alors de [6, Prop. 5.7]. 
Proposition 2.2. Soient $U$ et $V$ deux $k$-variétés géométriquement intègres telles que la $k$-variété $U \times_{k} V$ soit $k$-birationnelle à un espace projectif $\mathbf{P}_{k}^{n}$. Alors l'application naturelle $\operatorname{Br}(k) \rightarrow \mathrm{Br}_{n r}(k(U))$ est un isomorphisme.

Démonstration. L'hypothèse sur $U \times_{k} V$ et le fait que le corps $k$ soit infini assurent que les $k$-points lisses sont Zariski-denses sur $U$ et sur $V$. La projection

$$
U \times{ }_{k} V \rightarrow V
$$

induit une inclusion de corps $k \subset k(U) \subset k\left(U \times_{k} V\right) \simeq k\left(\mathbf{P}_{k}^{n}\right)$ et donc des homomorphismes

$$
\operatorname{Br}(k) \rightarrow \operatorname{Br}(k(U)) \rightarrow \operatorname{Br}\left(k\left(U \times_{k} V\right)\right)=\operatorname{Br}\left(k\left(\mathbf{P}^{n}\right)\right) .
$$

Ces homomorphismes sont injectifs. Ceci résulte par exemple du fait que les points $k$-rationnels sont Zariski-denses sur la $k$-variété $U$ et les points $k(U)$-rationnels sont Zariski-denses sur la $k(U)$-variété $V \times_{k} k(U)$. D'après [6, Lemma 5.4], ces homomorphismes induisent des homomorphismes

$$
\operatorname{Br}(k) \rightarrow \operatorname{Br}_{n r}(k(U)) \rightarrow \operatorname{Br}_{n r}\left(k\left(U \times_{k} V\right)\right)=\operatorname{Br}_{n r}\left(k\left(\mathbf{P}^{n}\right)\right)
$$

tous injectifs d'après ce qui précède. D'après [6, Prop. 5.7], l'application composée est un isomorphisme. Ceci établit la proposition.

\section{TORES ALGÉBRIQUeS ET INVARIANTS DE GROUPES ABÉLIENS}

La proposition suivante joue un rôle clé dans le présent article.

Proposition 3.1. Soit $k$ un corps et $T$ un $k$-tore déployé par une extension galoisienne finie $K / k$ de groupe de Galois un groupe métacyclique, i.e. dont tous les sous-groupes de Sylow sont cycliques. Il existe alors un $k$-tore $F$ tel que le produit $T \times_{k} F$ soit $k$-birationnel à un espace projectif sur $k$.

Démonstration. On sait (Voskresenskiı̌, Endo-Miyata; cf. [3]) que tout $k$-tore $T$ déployé par une extension galoisienne $K / k$ admet une résolution flasque, c'est-àdire qu'il existe une suite exacte de $k$-tores déployés par l'extension $K / k$

$$
1 \rightarrow F \rightarrow P \rightarrow T \rightarrow 1,
$$

où $P$ est un $k$-tore quasi-trivial, donc $k$-birationnel à un espace projectif et $F$ est un $k$-tore flasque (voir [3, $\S 1$, Lemme 3] et [3, $\S 5]$ ). D'après un théorème de Endo et Miyata (voir [3, Prop. 2, p. 184]), tout $k$-tore flasque $F$ déployé par une extension métacyclique est un facteur direct d'un $k$-tore quasi-trivial: il existe un $k$-tore $F^{\prime}$ tel que le $k$-tore $F \times{ }_{k} F^{\prime}$ soit $k$-isomorphe à un $k$-tore quasi-trivial. Ceci implique alors ([4, Prop. 7.4]) que le produit $T \times_{k} F$ est une $k$-variété $k$-rationnelle. On peut le voir directement de la façon suivante. La suite exacte de $k$-tores ci-dessus fait de $P$ un torseur sur $T$ sous $F$. La restriction de ce torseur au-dessus du point générique de $T$, donc sur le corps des fonctions $k(T)$ de la $k$-variété $T$, a une classe dans le groupe de cohomologie galoisienne $H^{1}(k(T), F)$. Comme $F$ est un facteur direct d'un tore quasi-trivial, le lemme de Shapiro et le théorème 90 de Hilbert sur tout corps, et en particulier sur toute extension finie du corps $k(T)$, impliquent $H^{1}(k(T), F)=0$. Le torseur sur $T$ sous $F$ défini par la suite exacte ci-dessus admet donc une section sur un ouvert non vide de $T$, ce qui implique que le produit $T \times{ }_{k} F$ est $k$-birationnel à la $k$-variété $P$, laquelle est un $k$-tore quasi-trivial, et donc est un ouvert d'un espace affine $\mathbf{A}_{k}^{N}$. 
L'énoncé suivant est une variante sur un corps non algébriquement clos d'un théorème de Fischer (cf. [6] Prop. 4.3]).

Proposition 3.2. Soit $G$ un groupe abélien fini, $G \hookrightarrow S L_{n, k}$ un plongement et $Y=S L_{n, k} / G$. Supposons la condition $\operatorname{Cyc}(G, k)$ satisfaite.

(i) Il existe une $k$-variété $Z$ telle que le produit $Y \times_{k} Z$ est $k$-birationnel à un espace projectif sur $k$.

(ii) La flèche naturelle $\operatorname{Br}(k) \rightarrow \mathrm{Br}_{n r}(k(Y))$ est un isomorphisme.

Démonstration. Si l'on a $G=G_{1} \times G_{2}$, un plongement $G_{1} \hookrightarrow X_{1}$, un plongement $G_{2} \hookrightarrow X_{2}$ et un plongement $G \hookrightarrow X$, où chacun des groupes $X$ et $X_{i}$ est un groupe spécial $k$-rationnel, alors

$$
X_{1} / G_{1} \times X_{2} / G_{2}=\left(X_{1} \times X_{2}\right) /\left(G_{1} \times G_{2}\right)=\left(X_{1} \times X_{2}\right) / G,
$$

et $\left(X_{1} \times X_{2}\right) / G$ est stablement $k$-birationnel à $X / G$ d'après le lemme 2.1

Pour établir la proposition, il suffit donc de considérer le cas où $G=\mathbf{Z} / p^{m}$ est un groupe abélien cyclique $p$-primaire, pour $p$ un nombre premier. L'extension $k\left(\mu_{p^{m}}\right) / k$ est cyclique si $p$ est impair, et c'est encore le cas si $p=2$ d'après l'hypothèse de la proposition.

Suivant Voskresenskiŭ, rappelons comment cela se traduit en termes de tores. Soit $g=\operatorname{Gal}\left(k\left(\mu_{p^{m}}\right) / k\right)$ et soit $\hat{G}=\operatorname{Hom}\left(G, \mu_{p^{m}}\right)=\mu_{p^{m}}$. Le groupe abélien libre de base les éléments de $\mu_{p^{m}}$, qui est un $g$-module de permutation, s'envoie de façon évidente et $g$-équivariante sur le groupe $\mu_{p^{m}}$. On note $\hat{T}_{G}$ le noyau de cette application. On a donc la suite exacte de $g$-modules continus discrets:

$$
0 \rightarrow \hat{T}_{G} \rightarrow \bigoplus_{\zeta \in \mu_{p} m} \mathbf{Z} . \zeta \rightarrow \hat{G} \rightarrow 0 .
$$

Par dualité, on obtient une suite exacte de $k$-groupes algébriques de type multiplicatif

$$
1 \rightarrow G \rightarrow P_{G} \rightarrow T_{G} \rightarrow 1,
$$

où $G=\mathbf{Z} / p^{m}, T_{G}$ est un $k$-tore et $P_{G}$ est un $k$-tore quasi-trivial, en particulier un $k$-groupe spécial $k$-rationnel. Ainsi ([6, Prop. 4.9]), pour tout $k$-plongement $G \hookrightarrow X=S L_{n, k}$, le quotient $X / G$ est stablement $k$-birationnel au $k$-tore $T_{G}$. Le $k$-tore $T_{G}$ est déployé par l'extension cyclique $k\left(\mu_{p^{m}}\right) / k$. La proposition 3.1 s'applique à $T=T_{G}$, ce qui établit l'énoncé (i) de la proposition 3.2. L'énoncé (ii) de cette proposition résulte alors de (i) et de la proposition 2.2

Remarque 3.3. Dans [12, Thm. 2.1], pour $G$ un groupe abélien, et sous l'hypothèse $C y c(G, k)$, D. Saltman a établi l'existence d'une "extension générique" sur $k$. La proposition 3.2 (i) donne une autre démonstration de son théorème.

\section{4. $G$-MOdules}

Soit $G$ un groupe fini. Pour tout $G$-module $M$ et tout entier $i \geq 0$, on définit les sous-groupes

$$
\amalg_{a b}^{i}(G, M) \subset \amalg_{b i c y c}^{i}(G, M) \subset \amalg_{c y c}^{i}(G, M) \subset H^{i}(G, M)
$$

comme les sous-groupes formés des éléments de $H^{i}(G, M)$ dont la restriction à chaque sous-groupe cyclique, resp. bicyclique, resp. abélien $H \subset G$ est nulle. Par définition, un groupe bicyclique est un groupe abélien engendré par deux éléments. Un $G$-module de permutation est un groupe abélien libre qui admet 
une base respectée par l'action de $G$. C'est une somme directe de $G$-modules $\operatorname{Hom}_{H}(\mathbf{Z}[G], \mathbf{Z}) \simeq \mathbf{Z}[G / H]$ pour divers sous-groupes $H \subset G$.

Lemme 4.1. Soit $G$ un groupe fini.

(i) Pour $M$ un $G$-module de permutation, on a $H^{1}(G, M)=0$.

(ii) Pour $M$ un $G$-module sans torsion, on a

$$
H^{1}(G, M \otimes \mathbf{Q} / \mathbf{Z}) \stackrel{\sim}{\longrightarrow} H^{2}(G, M)
$$

et, si l'action de $G$ est triviale, $H^{1}(G, M)=0$.

(iii) Pour $M$ un $G$-module avec action triviale, les groupes

$$
\amalg_{a b}^{1}(G, M) \subset \amalg_{b i c y c}^{1}(G, M) \subset \amalg_{c y c}^{1}(G, M)
$$

sont nuls.

(iv) Pour $M$ un $G$-module de permutation, les groupes

$$
\amalg_{a b}^{2}(G, M) \subset \amalg_{b i c y c}^{2}(G, M) \subset \amalg_{c y c}^{2}(G, M)
$$

sont nuls.

(v) Soit

$$
0 \rightarrow A \rightarrow B \rightarrow C \rightarrow 0
$$

une suite exacte de $G$-modules. Si $C$ est un $G$-module de permutation, cette suite induit des isomorphismes

$$
\begin{aligned}
\Psi_{a b}^{2}(G, A) & \stackrel{\simeq}{\rightarrow} \amalg_{a b}^{2}(G, B), \\
\amalg_{b i c y c}^{2}(G, A) & \stackrel{\simeq}{\rightarrow} \amalg_{b i c y c}^{2}(G, B), \\
\amalg_{c y c}^{2}(G, A) & \stackrel{\simeq}{\rightarrow} \amalg_{c y c}^{2}(G, B) .
\end{aligned}
$$

(vi) Soit

$$
0 \rightarrow A \rightarrow B \rightarrow C \rightarrow 0
$$

une suite exacte de $G$-modules. Si $C$ est un $G$-module sans torsion avec action triviale de $G$, cette suite induit des isomorphismes

$$
\begin{aligned}
\Psi_{a b}^{2}(G, A) & \stackrel{\simeq}{\rightarrow} \amalg_{a b}^{2}(G, B), \\
\amalg_{b i c y c}^{2}(G, A) & \stackrel{\simeq}{\rightarrow} \amalg_{b i c y c}^{2}(G, B), \\
\amalg_{c y c}^{2}(G, A) & \stackrel{\cong}{\rightarrow} \amalg_{c y c}^{2}(G, B) .
\end{aligned}
$$

Démonstration. Pour tout $G$-module $M$ avec action triviale de $G$, on a $H^{1}(G, M)=$ $\operatorname{Hom}(G, M)$. Ce groupe est nul si $M$ est de plus sans torsion. En particulier on a $H^{1}(G, \mathbf{Z})=\operatorname{Hom}(G, \mathbf{Z})=0$. Utilisant la suite exacte de $G$-modules triviaux

$$
0 \rightarrow \mathbf{Z} \rightarrow \mathbf{Q} \rightarrow \mathbf{Q} / \mathbf{Z} \rightarrow 0
$$

on obtient

$$
\amalg_{c y c}^{2}(G, \mathbf{Z}) \simeq \amalg_{c y c}^{1}(G, \mathbf{Q} / \mathbf{Z})=0,
$$

car tout caractère de $G$ nul sur tout élément de $G$ est nul.

Soit maintenant $K \subset H \subset G$ des sous-groupes. Pour tout $H$-module $A$, on définit le $G$-module coinduit $M_{H}^{G}(A)=\operatorname{Hom}_{H}(\mathbf{Z}[G], A)$ : voir [14, Chap. I, §2.5], où ce $G$-module est noté $M_{G}^{H}(A)$. Si $A$ est un $H$-module avec action trivale, alors on a un isomorphisme de $G$-modules

$$
\operatorname{Hom}_{H}(\mathbf{Z}[G], A) \simeq \mathbf{Z}[G / H] \otimes A .
$$


On a une flèche évidente $r: M_{H}^{G}(A) \rightarrow M_{K}^{G}(A)$. Pour tout entier $p$, et pour tout $G$-module $A$ avec action triviale, on a un diagramme commutatif

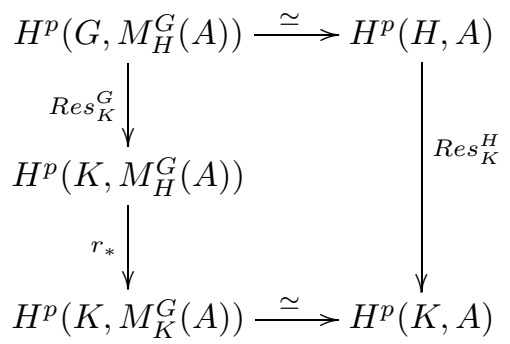

où les isomorphismes horizontaux sont donnés par le lemme de Shapiro.

De ceci on déduit d'une part $H^{1}(G, \mathbf{Z}[G / H]) \simeq H^{1}(H, \mathbf{Z})=0$, d'autre part, par considération de tous les sous-groupes cycliques $K \subset H$,

$$
\amalg_{c y c}^{2}(G, \mathbf{Z}[G / H]) \subset \amalg_{c y c}^{2}(H, \mathbf{Z})=0 .
$$

Ceci donne les énoncés (i) et (iv). Les autres énoncés en sont des conséquences formelles.

\section{Les RÉsultats}

Les notations sont celles de l'introduction. En particulier, $X=S L_{n, k}$, le groupe $G$ est un groupe fini vu comme $k$-groupe constant, $G \hookrightarrow S L_{n, k}$ est un $k$-plongement et $Y$ est l'espace homogène quotient $X / G$.

On note $\operatorname{Br}_{n r}^{0}(k(Y))$ le groupe de Brauer non ramifié normalisé, c'est-à-dire le groupe des éléments de $\operatorname{Br}_{n r}(k(Y)) \subset \operatorname{Br}(Y)$ qui s'annulent en l'image, par le $k$ morphisme $X \rightarrow Y$, du point $1 \in S L_{n}(k)=X(k)$.

Comme l'extension de corps $k(X) / k(Y)$ est galoisienne de groupe $G$, et que l'on a $H^{1}\left(G, k(X)^{\times}\right)=0$ d'après le théorème 90 de Hilbert, on a la suite exacte de restriction-inflation [15, X, $\S 4$, Corollaire de la Proposition 6]:

$$
0 \rightarrow H^{2}\left(G, k(X)^{\times}\right) \rightarrow \operatorname{Br}(k(Y)) \rightarrow \operatorname{Br}(k(X)) .
$$

On note $\left.H_{n r}^{2}\left(G, k(X)^{\times}\right)\right) \subset \operatorname{Br}(k(Y))$ le groupe $H^{2}\left(G, k(X)^{\times}\right) \cap \operatorname{Br}_{n r}(k(Y))$.

Proposition 5.1. (i) Dans $\operatorname{Br}(k(Y))$, on a $H^{2}\left(G, k(X)^{\times}\right) \cap \operatorname{Br}(k)=0$.

(ii) Le groupe $H_{n r}^{2}\left(G, k(X)^{\times}\right) \subset H^{2}\left(G, k(X)^{\times}\right) \subset \operatorname{Br}(k(Y))$ coincide avec le groupe $\mathrm{Br}_{n r}^{0}(k(Y))$.

(iii) Le groupe $H_{n r}^{2}\left(G, k(X)^{\times}\right)=\operatorname{Br}_{n r}^{0}(k(Y))$ est fini.

Démonstration. Comme $X(k) \neq \emptyset$ et donc $Y(k) \neq \emptyset$, on a des inclusions naturelles compatibles $\operatorname{Br}(k) \hookrightarrow \operatorname{Br}_{n r}(k(Y))$ et $\operatorname{Br}(k) \hookrightarrow \operatorname{Br}_{n r}(k(X))$. Comme $H^{2}\left(G, k(X)^{\times}\right)$ a une image nulle dans $\operatorname{Br}(k(X))$, l'intersection de $H^{2}\left(G, k(X)^{\times}\right)$et de $\operatorname{Br}(k)$ dans $\operatorname{Br}(k(Y))$ est réduite à 0 . Ceci établit (i). Par définition,

$$
H_{n r}^{2}\left(G, k(X)^{\times}\right)=H^{2}\left(G, k(X)^{\times}\right) \cap \operatorname{Br}_{n r}(k(Y)) \subset \operatorname{Br}(k(Y)) .
$$

Une classe dans ce groupe a une image triviale dans $\operatorname{Br}(k(X))$ et donc une image triviale par évaluation au point image de $1 \in X(k)$. On a donc

$$
H_{n r}^{2}\left(G, k(X)^{\times}\right) \subset \operatorname{Br}_{n r}^{0}(k(Y)) .
$$

Par ailleurs, l'image de tout élément de $\operatorname{Br}_{n r}^{0}(k(Y)) \subset \operatorname{Br}(k(Y))$ dans $\operatorname{Br}_{n r}(k(X))$ est une classe d'une part triviale en $1 \in X(k)$ d'autre part constante car $X$ étant 
une variété $k$-rationnelle, l'application $\operatorname{Br}(k) \hookrightarrow \mathrm{Br}_{n r}(k(X))$ est un isomorphisme [6. Prop. 5.7]. Ainsi $\operatorname{Br}_{n r}^{0}(k(Y))$ est dans le noyau de la flèche de restriction $\operatorname{Br}(k(Y)) \rightarrow \operatorname{Br}(k(X))$, donc $\operatorname{Br}_{n r}^{0}(k(Y)) \subset H^{2}\left(G, k(X)^{\times}\right)$d'après la suite de restriction-inflation citée ci-dessus, et donc $\operatorname{Br}_{n r}^{0}(k(Y)) \subset H_{n r}^{2}\left(G, k(X)^{\times}\right)$. Tout ceci établit (ii).

L'énoncé de finitude (iii) vaut plus généralement pour les corps de fonctions de variétés projectives, lisses, géométriquement connexes qui sont géométriquement unirationnelles. Rappelons la démonstration. Pour toute $k$-variété projective, lisse, géométriquement intègre $Z$, notant $\bar{Z}=Z \times_{k} \bar{k}$, la suite spectrale de Leray pour le morphisme $Z \rightarrow$ Spec $k$, la topologie étale, et le faisceau $\mathbb{G}_{m}$ sur $Z$ donne naissance à une suite exacte

$$
\operatorname{Br}(k) \rightarrow \operatorname{Ker}[\operatorname{Br}(Z) \rightarrow \operatorname{Br}(\bar{Z})] \rightarrow H^{1}(\mathfrak{g}, \operatorname{Pic}(\bar{Z})) .
$$

Voir par exemple [5, (1.5.0), p. 386] ou [16, (2.23), p. 30]. Sous l'hypothèse car. $(k)=0$, si $Z$ est géométriquement unirationnelle, sa variété de Picard est triviale, ce qui implique que le module galoisien $\operatorname{Pic}(\bar{Z})$ coïncide avec le groupe de Néron-Severi de $\bar{Z}$, donc est un groupe de type fini. Comme de plus $Z$ est géométriquement unirationnelle, par un théorème de Serre [13, Prop. 1], son groupe fondamental géométrique est nul, et $\operatorname{donc} \operatorname{Pic}(\bar{Z})$ est sans torsion. Ainsi $\operatorname{Pic}(\bar{Z})$ est un groupe abélien libre de type fini et donc le groupe $H^{1}(\mathfrak{g}, \operatorname{Pic}(\bar{Z}))$ est fini. Par hypothèse, il existe une application rationnelle dominante génériquement finie de $\mathbf{P}_{\bar{k}}^{d}$ vers $\bar{Z}$, où $d$ est la dimension de $\bar{Z}$. Ceci définit une extension finie de corps $\bar{k}\left(\mathbf{P}^{d}\right) / \bar{k}(Z)$ de degré disons $n>0$. Par un argument bien connu (lemme 5.2 ci-dessous) on en déduit que l'homomorphisme $\operatorname{Br}\left((\bar{k}(Z)) \rightarrow \operatorname{Br}\left(\bar{k}\left(\mathbf{P}^{d}\right)\right)\right.$ a son noyau annulé par $n$. Cet homomorphisme envoie $\operatorname{Br}(\bar{Z})=\operatorname{Br}_{n r}(\bar{k}(Z))$ dans $\operatorname{Br}_{n r}\left(\bar{k}\left(\mathbf{P}^{d}\right)\right)=0$. Ainsi $\operatorname{Br}(\bar{Z})$ est annulé par un entier positif $n$. Une application de la suite de Kummer en cohomologie étale ([8, II, §3]) montre que le groupe de $n$-torsion $\operatorname{Br}(\bar{Z})[n]$ est un quotient du groupe de cohomologie étale $H_{\text {ét }}^{2}\left(\bar{Z}, \mu_{n}\right)$, et on sait bien que ce groupe est fini. Donc $\operatorname{Br}(\bar{Z})$ est fini, et donc finalement le quotient de $\operatorname{Br}(Z)$ par l'image de $\operatorname{Br}(k)$ est fini. L'hypothèse car. $(k)=0$ garantit (Hironaka) l'existence d'une $k$-compactification lisse $Z$ de la $k$-variété lisse $Y=S L_{n, k} / G$. On a (cf. [2]) $\operatorname{Br}(Z)=\operatorname{Br}_{n r}(k(Y))$, et $\operatorname{Br}(Z) / \operatorname{Br}(k)=\operatorname{Br}_{n r}(k(Y)) / \operatorname{Br}(k) \simeq \operatorname{Br}_{n r}^{0}(k(Y))$. Ainsi $\mathrm{Br}_{n r}^{0}(k(Y))$ est fini.

On a utilisé le lemme bien connu suivant.

Lemme 5.2. Soit $F / E$ une extension séparable de corps de degré $n$. L'application induite $\operatorname{Br}(E) \rightarrow \operatorname{Br}(F)$ sur les groupes de Brauer a son noyau annulé par $n$.

Démonstration. Soit $E_{s} / E$ une clôture séparable contenant le corps $F$. Soit $G=$ $\operatorname{Gal}\left(E_{s} / E\right)$ et $H \subset G$ le sous-groupe ouvert $H=\operatorname{Gal}\left(E_{s} / F\right)$ du groupe profini $G$. Une application de [14, Chap. I, §2.4, Prop. 9], au module galoisien continu discret $A=E_{s}^{\times}$et à $q=2$, montre que le noyau de la flèche de restriction $H^{2}\left(G, E_{s}^{\times}\right) \rightarrow$ $H^{2}\left(H, E_{s}^{\times}\right)$est annulé par $n$. Or cette application n'est autre que la flèche de restriction $\operatorname{Br}(E) \rightarrow \operatorname{Br}(F)$ ([15, Chap. X]).

Notons que dans la décomposition d'un élément non ramifié de

$$
H^{2}\left(G, k(X)^{\times}\right) \subset \operatorname{Br}(k(Y))
$$

en ses composantes $p$-primaires pour chaque premier $p$, chacune de celles-ci est non ramifiée. 
Théorème 5.3. (i) Supposons le groupe $G$ abélien. Si la condition $C y c(G, k)$ est satisfaite, alors $\mathrm{Br}_{n r}^{0}(k(Y))=0$.

(ii) Supposons le groupe $G$ abélien. On a $2 \cdot \operatorname{Br}_{n r}^{0}(k(Y))=0$.

Soit $G$ fini quelconque.

(iii) Pour tout sous-groupe abélien $A \subset G$, l'image de $H_{n r}^{2}\left(G, k(X)^{\times}\right)$dans $H^{2}\left(A, k(X)^{\times}\right)$est annulée par 2 .

(iv) Si la condition $C y c(G, k)$ est satisfaite, pour tout sous-groupe abélien $A \subset G$, l'image de $H_{n r}^{2}\left(G, k(X)^{\times}\right)$dans $H^{2}\left(A, k(X)^{\times}\right)$est nulle.

Démonstration. L'énoncé (i) n'est qu'une reformulation du point (ii) de la proposition 3.2. Soit $K=k(\sqrt{-1})$. La condition $C y c(G, K)$ est satisfaite. On conclut $\operatorname{Br}_{n r}^{0}(K(Y))=0$. Par corestriction-restriction de $k$ à $K$, ceci implique

$$
2 . \operatorname{Br}_{n r}^{0}(k(Y))=0 \text {, }
$$

c'est-à-dire (ii). Les énoncés (iii) et (iv) résultent alors simplement de (i) et (ii) et $\mathrm{du}$ fait que pour tout sous-groupe $H \subset G$, la flèche de restriction $H^{2}\left(G, k(X)^{\times}\right) \rightarrow$ $H^{2}\left(H, k(X)^{\times}\right)$envoie le sous-groupe $H_{n r}^{2}\left(G, k(X)^{\times}\right) \subset \mathrm{Br}_{n r}\left(k(X)^{G}\right)$ dans le sousgroupe $H_{n r}^{2}\left(H, k(X)^{\times}\right) \subset \operatorname{Br}_{n r}\left(k(X)^{H}\right)$ (voir [6, Lemma 5.5]).

Remarque 5.4. La proposition 3.2 implique aussi un énoncé sur la cohomologie non ramifiée de degré quelconque (cf. [2]). Soient $i \geq 0$ et $m \geq 1$ des entiers, $j \in \mathbf{Z}$. Alors:

(i) Tout élément normalisé de $H_{n r}^{i}\left(k(X)^{G}, \mu_{m}^{\otimes j}\right)$ a une image nulle dans le groupe $H^{i}\left(k(X)^{A}, \mu_{m}^{\otimes j}\right)$ pour tout sous-groupe abélien $A \subset G$ d'ordre impair.

(ii) Si la condition $\operatorname{Cyc}(G, k)$ est satisfaite, tout élément normalisé du groupe $H_{n r}^{i}\left(k(X)^{G}, \mu_{m}^{\otimes j}\right)$ a une image nulle dans $H^{i}\left(k(X)^{A}, \mu_{m}^{\otimes j}\right)$ pour tout sous-groupe abélien $A \subset G$.

Théorème 5.5. Supposons la condition $C y c(G, k)$ satisfaite. Soit $\mu(k)$ le groupe des racines de l'unité dans $k$.

(a) La flèche naturelle

$$
H^{2}\left(G, k^{\times}\right) \rightarrow H^{2}\left(G, k(X)^{\times}\right)
$$

induit un isomorphisme entre un sous-groupe de $\amalg_{a b}^{2}\left(G, k^{\times}\right)$et $H_{n r}^{2}\left(G, k(X)^{\times}\right)$.

(b) La flèche $H^{2}(G, \mu(k)) \rightarrow H^{2}\left(G, k^{\times}\right)$induit un isomorphisme de groupes

$$
\amalg_{a b}^{2}(G, \mu(k)) \stackrel{\simeq}{\rightarrow} \amalg_{a b}^{2}\left(G, k^{\times}\right),
$$

et la flèche composée

$$
H^{2}(G, \mu(k)) \rightarrow H^{2}\left(G, k^{\times}\right) \rightarrow H^{2}\left(G, k(X)^{\times}\right)
$$

identifie un sous-groupe du groupe $\amalg_{a b}^{2}(G, \mu(k))$ avec le groupe

$$
\operatorname{Br}_{n r}^{0}(k(Y)) \subset H^{2}\left(G, k(X)^{\times}\right) .
$$

Démonstration. Toute fonction inversible sur le groupe $X=S L_{n, k}$ est constante, et le groupe de Picard de $S L_{n, k}$ est nul. La flèche diviseur $k(X)^{\times} \rightarrow \operatorname{Div}(X)$ sur le corps des fonctions de $X$ donne donc naissance à une suite exacte de $G$-modules

$$
1 \rightarrow k^{\times} \rightarrow k(X)^{\times} \rightarrow \operatorname{Div}(X) \rightarrow 0 .
$$

Le $G$-module $\operatorname{Div}(X)$ est le groupe abélien libre sur les points de codimension 1 de $X$. C'est donc un $G$-module de permutation. 
Le lemme 4.1 (v) donne alors

$$
\amalg_{a b}^{2}\left(G, k^{\times}\right) \stackrel{\sim}{\rightarrow} \amalg_{a b}^{2}\left(G, k(X)^{\times}\right) .
$$

D'après le théorème 5.3 , on a une injection

$$
H_{n r}^{2}\left(G, k(X)^{\times}\right) \hookrightarrow \amalg_{a b}^{2}\left(G, k(X)^{\times}\right),
$$

ce qui établit (a).

Pour tout corps $k$ on a la suite exacte de $G$-modules (avec action triviale)

$$
1 \rightarrow \mu(k) \rightarrow k^{\times} \rightarrow k^{\times} / \mu(k) \rightarrow 1
$$

où $R:=k^{\times} / \mu(k)$ est sans torsion. D'après le lemme 4.1 (vi), on a

$$
\amalg_{a b}^{2}(G, \mu(k)) \stackrel{\simeq}{\rightrightarrows} \amalg_{a b}^{2}\left(G, k^{\times}\right) .
$$

L'énoncé (b) résulte alors de l'énoncé (a) et de la proposition 5.1(ii).

Remarque 5.6. Sous l'hypothèse $C y c(G, k)$, cet argument donne une autre démonstration de la finitude de $\operatorname{Br}_{n r}^{0}(k(Y))$ (proposition 5.1). Il suffit d'observer que le groupe $H^{2}(G, \mu(k))$, et donc aussi $\amalg_{a b}^{2}(G, \mu(k)) \subset H^{2}(G, \mu(k))$, est fini. Soit $l$ premier divisant l'ordre de $G$. Le groupe $\mu(k)\{l\}$ est soit fini soit isomorphe à $\mathbf{Q}_{l} / \mathbf{Z}_{l}$. Dans les deux cas, le groupe $H^{2}(G, \mu(k)\{l\})$ est fini. C'est clair dans le premier cas. Dans le second, $H^{2}\left(G, \mathbf{Q}_{l} / \mathbf{Z}_{l}\right) \stackrel{\sim}{\longrightarrow} H^{3}(G, \mathbf{Z})\{l\}$. Si l'on ne fait pas l'hypothèse $C y c(G, k)$, cet argument donne au moins la finitude du sous-groupe $\bigoplus_{l \neq 2} \operatorname{Br}_{n r}^{0}(k(Y))\{l\}$ (torsion impaire du groupe de torsion $\operatorname{Br}_{n r}^{0}(k(Y))$ ).

Corollaire 5.7. Soit $\mu(k)$ le groupe des racines de l'unité dans $k$.

(a) Si le groupe $\mu(k)$ est fini et d'ordre premier à celui de $G$ (ce qui implique que l'ordre de $G$ est impair et $C y c(G, k)$ satisfaite $)$, alors $\operatorname{Br}_{n r}^{0}(k(Y))=0$.

(b) Pour $k=\mathbf{Q}$ et $G$ d'ordre impair, on a $\operatorname{Br}(\mathbf{Q})=\operatorname{Br}_{n r}(\mathbf{Q}(Y))$.

(c) Soit $k=\mathbf{R}$.

(c1) On a $\operatorname{Br}_{n r}^{0}(\mathbf{R}(Y)) \subset \amalg_{a b}^{2}(G, \mathbf{Z} / 2)$.

(c2) On a $2 . \mathrm{Br}_{n r}(\mathbf{R}(Y))=0$.

(c3) Si les 2-sous-groupes de Sylow de $G$ sont abéliens, alors

$$
\operatorname{Br}_{n r}^{0}(\mathbf{R}(Y))=0 .
$$

Démonstration. Dans le cas (a), on a $H^{2}(G, \mu(k))=0$, car ce groupe est annulé par l'ordre de $G$ et par l'ordre de $\mu(k)$. Le théorème 5.5 donne alors (a), et (b) est alors une conséquence immédiate, puisque $\mu(\mathbf{Q})=\mathbf{Z} / 2$. Sur $\mathbf{R}$, la condition $C y c(G, \mathbf{R})$ est trivialement satisfaite, et $\mu(\mathbf{R})=\mathbf{Z} / 2$. Ceci donne (c1) et, comme conséquence triviale, (c2). Un argument de corestriction-restriction bien connu (remarque entre crochets suivant [14, Chap. I, §2.4, Corollaire de la Proposition 9]) montre que si les 2-groupes de Sylow de $G$ sont abéliens, alors le groupe abélien $\amalg_{a b}^{2}(G, \mathbf{Z} / 2)$ est annulé par un entier impair. Comme ce groupe est clairement annulé par 2, il est nul. Compte tenu de (c1), on obtient (c3).

Remarque 5.8. Dans la situation du (a) du corollaire 5.7 ci-dessus, sur un corps de nombres, par une méthode arithmétique, Demarche [7. Thm. 5] a établi la nullité du groupe $\mathrm{Br}_{n r, 1}^{0}(k(Y)) \subset \mathrm{Br}_{n r}^{0}(k(Y))$. 
Remarque 5.9. Comme me l'a indiqué C. Demarche, les p-groupes qu'il étudie dans [7. §6] fournissent, pour $p=2$, un exemple de groupe fini $G$ d'ordre 64 tel que le groupe de Brauer non ramifié normalisé $\mathrm{Br}_{n r}^{0}(\mathbf{Q}(Y))$ possède un élément algébrique (i.e. nul dans $\operatorname{Br}(\overline{\mathbf{Q}}(Y)))$ qui ne s'annule pas dans $\operatorname{Br}_{n r}^{0}(\mathbf{R}(Y))$.

L'isomorphisme $\amalg_{b i c y c}^{2}\left(G, k^{\times}\right) \stackrel{\sim}{\rightarrow} H_{n r}^{2}\left(G, k(X)^{\times}\right)$dans l'énoncé suivant, généralisation du théorème de Bogomolov pour $k=\bar{k}$, a déjà été obtenu par Nguyen Thi Kim Ngan dans sa thèse [11, Thm. 3.2.2].

Théorème 5.10. Supposons que pour tout groupe cyclique $\mathbf{Z} / m \subset G$, on a l'égalité $\mu_{m}(k)=\mu_{m}(\bar{k})$. On a alors des isomorphismes de groupes finis

$$
\amalg_{a b}^{2}\left(G, k^{\times}\right) \stackrel{\widetilde{\sim}}{\rightarrow} \amalg_{b i c y c}^{2}\left(G, k^{\times}\right) \stackrel{\simeq}{\rightarrow} H_{n r}^{2}\left(G, k(X)^{\times}\right)
$$

et

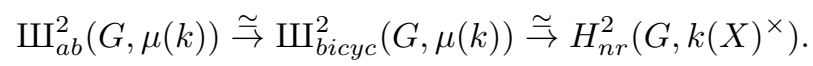

Démonstration. L'hypothèse faite sur les racines de l'unité implique que $C y c(G, k)$ est satisfaite.

D'après le théorème 5.3, on a les inclusions

$$
H_{n r}^{2}\left(G, k(X)^{\times}\right) \subset \amalg_{a b}^{2}\left(G, k(X)^{\times}\right) \subset \amalg_{b i c y c}^{2}\left(G, k(X)^{\times}\right) .
$$

L'hypothèse faite sur les racines de l'unité implique aussi que pour tout anneau de valuation discrète de rang 1 de $k(X)^{G}$, contenant $k$, l'action des sous-groupes de décomposition de $G$ sur l'inertie est triviale. On peut donc copier l'argument de Bogomolov (cf. [6. Thm. 6.1, Thm. 7.1]), qui mène à l'inclusion

$$
\amalg_{b i c y c}^{2}\left(G, k(X)^{\times}\right) \subset H_{n r}^{2}\left(G, k(X)^{\times}\right) .
$$

Dans $H^{2}\left(G, k(X)^{\times}\right)$, on a donc les égalités

$$
\amalg_{a b}^{2}\left(G, k(X)^{\times}\right)=\amalg_{b i c y c}^{2}\left(G, k(X)^{\times}\right)=H_{n r}^{2}\left(G, k(X)^{\times}\right) .
$$

Comme dans la démonstration du théorème [5.5, on déduit du lemme 4.1 les isomorphismes

$$
\begin{aligned}
& \amalg_{a b}^{2}\left(G, k^{\times}\right) \stackrel{\widetilde{\sim}}{\rightarrow} \amalg_{a b}^{2}\left(G, k(X)^{\times}\right) \\
& \amalg_{b i c y c}^{2}\left(G, k^{\times}\right) \stackrel{\simeq}{\rightarrow} \amalg_{b i c y c}^{2}\left(G, k(X)^{\times}\right) \\
& \amalg_{b i c y c}^{2}(G, \mu(k)) \stackrel{\simeq}{\rightarrow} \amalg_{b i c y c}^{2}\left(G, k^{\times}\right) \\
& \amalg_{a b}^{2}(G, \mu(k)) \stackrel{\widetilde{\sim}}{\rightarrow} \amalg_{a b}^{2}\left(G, k^{\times}\right) .
\end{aligned}
$$

Notons $\hat{G}=\operatorname{Hom}(G, \mathbf{Q} / \mathbf{Z})$ le groupe des caractères du groupe $G$.

Théorème 5.11. Supposons que le corps $k$ ne contient qu'un nombre fini de racines de l'unité, et supposons la condition $C y c(G, k)$ satisfaite.

(i) Soit $r>0$ tel que $\mu(k)=\mu_{r}(\bar{k})$. Le groupe de Brauer non ramifié algébrique normalisé

$$
\operatorname{Ker}\left[\operatorname{Br}_{n r}^{0}\left(k(X)^{G}\right) \rightarrow \operatorname{Br}\left(\bar{k}(X)^{G}\right)\right]=\operatorname{Ker}\left[H_{n r}^{2}\left(G, k(X)^{\times}\right) \rightarrow H^{2}\left(G, \bar{k}(X)^{\times}\right)\right]
$$

s'identifie à un sous-groupe de $\operatorname{Ker}\left[\hat{G} / r \rightarrow \prod_{A} \hat{A} / r\right]$, où $A$ parcourt les sous-groupes abéliens de $G$.

(ii) Si $G$ est un groupe simple non abélien, on a $\operatorname{Br}_{n r}^{0}\left(k(X)^{G}\right)=0$. 
Démonstration. Le théorème 5.5 donne un plongement

$$
\operatorname{Br}_{n r}^{0}\left(k(X)^{G}\right) \hookrightarrow \amalg_{a b}^{2}(G, \mu(k)) .
$$

Par ailleurs, sur $\bar{k}$, d'après Bogomolov (Thm. [5.10 ci-dessus) on a un isomorphisme

$$
\mathrm{Br}_{n r}^{0}\left(\bar{k}(X)^{G}\right) \stackrel{\widetilde{\sim}}{\rightarrow} \amalg_{a b}^{2}(G, \mu(\bar{k})) .
$$

Les flèches $\operatorname{Br}_{n r}^{0}\left(k(X)^{G}\right) \rightarrow H^{2}(G, \mu(k))$ et $\operatorname{Br}_{n r}^{0}\left(\bar{k}(X)^{G}\right) \rightarrow H^{2}(G, \mu(\bar{k}))$ sont compatibles, comme on le vérifie immédiatement. On a donc un plongement

$$
\operatorname{Ker}\left[\operatorname{Br}_{n r}^{0}\left(k(X)^{G}\right) \rightarrow \operatorname{Br}_{n r}^{0}\left(\bar{k}(X)^{G}\right)\right] \hookrightarrow \operatorname{Ker}\left[H^{2}(G, \mu(k)) \rightarrow H^{2}(G, \mu(\bar{k}))\right],
$$

et donc un plongement

$$
\operatorname{Ker}\left[\operatorname{Br}_{n r}^{0}\left(k(X)^{G}\right) \rightarrow \operatorname{Br}_{n r}^{0}\left(\bar{k}(X)^{G}\right)\right] \hookrightarrow \operatorname{Ker}\left[\amalg_{a b}^{2}(G, \mu(k)) \rightarrow \amalg_{a b}^{2}(G, \mu(\bar{k}))\right] .
$$

On a la suite exacte de $G$-modules avec action triviale

$$
1 \rightarrow \mu(k) \rightarrow \mu(\bar{k}) \stackrel{x \mapsto x^{r}}{\longrightarrow} \mu(\bar{k}) \rightarrow 1 .
$$

Fixons un isomorphisme $\mathbf{Q} / \mathbf{Z} \stackrel{\sim}{\rightarrow} \mu(\bar{k})$. Pour tout sous-groupe $H \subset G$, de groupe des caractères $\hat{H}=\operatorname{Hom}(H, \mathbf{Q} / \mathbf{Z})$, en prenant la $H$-cohomologie de la suite exacte ci-dessus, on obtient une suite exacte

$$
0 \rightarrow \hat{H} / r \rightarrow H^{2}(H, \mu(k)) \rightarrow H^{2}(H, \mu(\bar{k})) .
$$

Passant du groupe $G$ à l'ensemble de ses sous-groupes abéliens $A$, on obtient un diagramme commutatif de suites exactes:

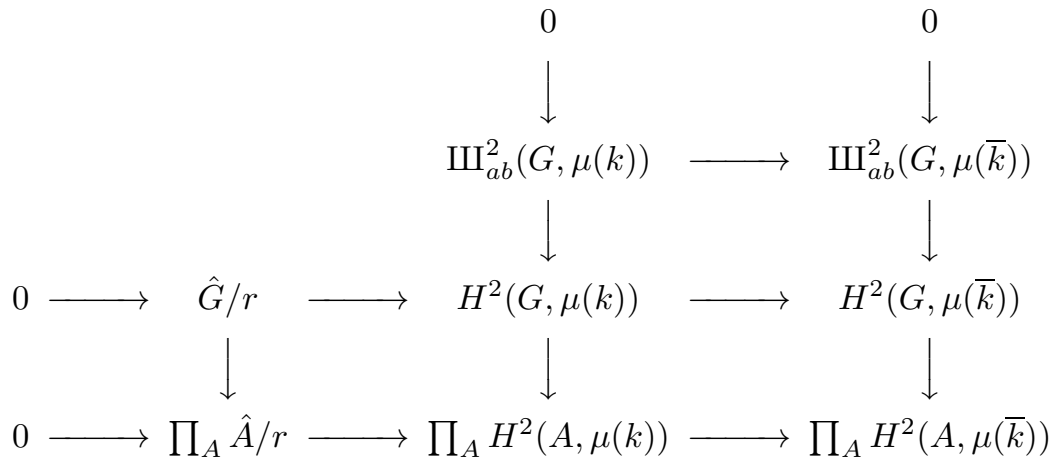

d'où l'on déduit un isomorphisme

$$
\operatorname{Ker}\left[\amalg_{a b}^{2}(G, \mu(k)) \rightarrow \amalg_{a b}^{2}(G, \mu(\bar{k}))\right] \simeq \operatorname{Ker}\left[\hat{G} / r \rightarrow \prod_{A} \hat{A} / r\right] .
$$

Ceci établit l'énoncé (i).

Si $G$ est comme en (ii), alors d'une part $\hat{G}=0$, d'autre part

$$
\operatorname{Br}_{n r}^{0}\left(\bar{k}(X)^{G}\right)=0,
$$

d'après Kunyavskiı̌ [10]. L'énoncé (ii) résulte donc de (i).

Remarque 5.12. Le théorème 5.11] s'applique aux corps de nombres et plus généralement aux corps de type fini sur un corps de nombres, mais aussi aux corps $p$-adiques et aussi aux réels. Sur de tels corps, on voit donc que si le groupe $G$ n'a pas de caractères, le groupe de Brauer non ramifié algébrique normalisé est nul. 
Sur un corps de nombres, ceci avait été établi par Harari [9, Prop. 4], qui donne une formule pour le groupe de Brauer non ramifié algébrique normalisé comme sousgroupe de $H^{1}(\mathfrak{g}, \operatorname{Hom}(G, \mathbf{Q} / \mathbf{Z}(1)))$, formule qui implique clairement que ce groupe est nul si $G$ n'a pas de caractères.

Pour les réels, on obtient l'énoncé suivant.

Corollaire 5.13. Soit $k=\mathbf{R}$. Le groupe de Brauer non ramifié algébrique normalisé de $\mathbf{R}(X)^{G}$

$$
\operatorname{Ker}\left[\operatorname{Br}_{n r}^{0}\left(\mathbf{R}(X)^{G}\right) \rightarrow \operatorname{Br}\left(\mathbf{C}(X)^{G}\right)\right]
$$

s'identifie à un sous-groupe de $\operatorname{Ker}\left[\hat{G} / 2 \rightarrow \prod_{A} \hat{A} / 2\right]$, où A parcourt les sous-groupes abéliens de $G$.

\section{REFERENCES}

[1] F. A. Bogomolov, The Brauer group of quotient spaces of linear representations (Russian), Izv. Akad. Nauk SSSR Ser. Mat. 51 (1987), no. 3, 485-516, 688; English transl., Math. USSR-Izv. 30 (1988), no. 3, 455-485. MR903621 (88m:16006)

[2] J.-L. Colliot-Thélène, Birational invariants, purity and the Gersten conjecture, Division algebras (Santa Barbara, CA, 1992), Proc. Sympos. Pure Math., vol. 58, Amer. Math. Soc., Providence, RI, 1995, pp. 1-64. MR1327280 (96c:14016)

[3] Jean-Louis Colliot-Thélène et Jean-Jacques Sansuc, La R-équivalence sur les tores, Ann. Sci. École Norm. Sup. (4) 10 (1977), no. 2, 175-229. MR0450280 (56 \#8576)

[4] Jean-Louis Colliot-Thélène et Jean-Jacques Sansuc, Principal homogeneous spaces under flasque tori: applications, J. Algebra 106 (1987), no. 1, 148-205, DOI 10.1016/00218693(87)90026-3. MR878473 (88j:14059)

[5] Jean-Louis Colliot-Thélène et Jean-Jacques Sansuc, La descente sur les variétés rationnelles. II, Duke Math. J. 54 (1987), no. 2, 375-492, DOI 10.1215/S0012-7094-87-05420-2. MR899402 (89f:11082)

[6] Jean-Louis Colliot-Thélène et Jean-Jacques Sansuc, The rationality problem for fields of invariants under linear algebraic groups (with special regards to the Brauer group), Algebraic groups and homogeneous spaces, Tata Inst. Fund. Res. Stud. Math., Tata Inst. Fund. Res., Mumbai, 2007, pp. 113-186. MR2348904 (2008m:13010)

[7] Cyril Demarche, Groupe de Brauer non ramifié d'espaces homogènes à stabilisateurs finis, Math. Ann. 346 (2010), no. 4, 949-968, DOI 10.1007/s00208-009-0415-8. MR2587098 (2011g:14052)

[8] A. Grothendieck, Le groupe de Brauer I, II, III, in Dix exposés sur la cohomologie des schémas, North-Holland, Amsterdam; Masson, Paris, 1968. MR.0244269 (39 \#5586a), MR0244270 (39 \#5586b) MR0244271 (39 \#5586c)

[9] David Harari, Quelques propriétés d'approximation reliées à la cohomologie galoisienne d'un groupe algébrique fini, Bull. Soc. Math. France 135 (2007), no. 4, 549-564. MR2439198 (2009f:11041)

[10] Boris Kunyavskiı̌, The Bogomolov multiplier of finite simple groups, Cohomological and geometric approaches to rationality problems, Progr. Math., vol. 282, Birkhäuser Boston Inc., Boston, MA, 2010, pp. 209-217, DOI 10.1007/978-0-8176-4934-0_8. MR2605170 (2011b:20033)

[11] Nguyen Thi Kim Ngan, Thèse, Université Paris VII, 2010.

[12] David J. Saltman, Generic Galois extensions and problems in field theory, Adv. in Math. 43 (1982), no. 3, 250-283, DOI 10.1016/0001-8708(82)90036-6. MR648801 (84a:13007)

[13] J.-P. Serre, On the fundamental group of a unirational variety, J. London Math. Soc. 34 (1959), 481-484. MR0109155 (22 \#43)

[14] Jean-Pierre Serre, Cohomologie galoisienne, Cinquième édition, révisée et complétée, Lecture Notes in Mathematics, vol. 5, Springer-Verlag, Berlin, 1994. MR1324577 (96b:12010) 
[15] Jean-Pierre Serre, Corps locaux, Deuxième édition, Publications de l'Université de Nancago, No. VIII, Hermann, Paris, 1968. MR0354618 (50 \#7096)

[16] Alexei Skorobogatov, Torsors and rational points, Cambridge Tracts in Mathematics, vol. 144, Cambridge University Press, Cambridge, 2001. MR.1845760 (2002d:14032)

CNRS, Université Paris-Sud, Département de mathématiques, BÂtiment 425, 91405 Orsay Cedex, France

E-mail address: jlct@math.u-psud.fr 